PROCEEDINGS OF THE

AMERICAN MATHEMATICAL SOCIETY

Volume 127, Number 7, Pages 2193-2197

S 0002-9939(99)04737-1

Article electronically published on March 1, 1999

\title{
NORMALITY AND PARACOMPACTNESS OF THE FELL TOPOLOGY
}

\author{
L'. HOLÁ, S. LEVI, AND J. PELANT \\ (Communicated by Alan Dow)
}

\begin{abstract}
Let $X$ be a Hausdorff topological space and $C L(X)$ the hyperspace of all closed nonempty subsets of $X$. We show that the Fell topology on $C L(X)$ is normal if and only if the space $X$ is Lindelöf and locally compact. For the Fell topology normality, paracompactness and Lindelöfness are equivalent.
\end{abstract}

Throughout the paper all spaces are assumed to be Hausdorff. By $X$ we always denote a space, while $C L(X)$ (resp. $K(X)$ ) is the set of all nonempty closed (compact) subsets of $X$. We quote [En] and [Be1] for the basic notions. One of the most important and well-studied hyperspace topologies on $C L(X)$ is the Fell topology [At], [Be1], [Be2], [Fe], [Fl], [Po]. The Fell topology can be considered a classical one, as it has found numerous applications in different fields of mathematics ([Ma], $[\mathrm{At}])$.

To describe this topology, we need to introduce some notation. For $E$ a subset of $X$, we associate the following subsets of $C L(X)$ :

$$
\begin{aligned}
& E^{-}=\{A \in C L(X): A \cap E \neq \emptyset\}, \\
& E^{+}=\{A \in C L(X): A \subset E\} .
\end{aligned}
$$

The Fell topology $\tau_{F}$ on $C L(X)$ has as a subbase all sets of the form $V^{-}$, where $V$ is an open subset of $X$ plus all sets of the form $\left(K^{c}\right)^{+}$, where $K \in K(X)$ and $K^{c}$ is the complement of $K$.

In locally compact spaces, convergence with respect to the Fell topology is $\mathrm{Ku}$ ratowski convergence of nets of sets.

If compact subsets in the above definition are replaced by closed sets, we obtain the stronger Vietoris topology, also called the finite topology [Mi]. The normality of the Vietoris topology on $C L(X)$ is equivalent to the compactness of $X$ as was shown by Veličko in [Ve]. We refer also to Keesling's deep study of normality of the Vietoris topology [Ke1], [Ke2]. The regularity and Hausdorffness of the Fell topology was studied by Poppe [Po] and the complete regularity by Beer and Tamaki in [BT]. We can summarize here these results as follows:

Received by the editors February 12, 1997 and, in revised form, October 7, 1997.

1991 Mathematics Subject Classification. Primary 54B20.

Key words and phrases. Fell topology, locally compact Hausdorff space, Lindelöf space, normal space, $\sigma$-compact.

The third author was partially supported by the grant GACR 201/94/0069 and the grant 119 401 of Acad. Sci. CR. 
Proposition A ([BT]). Let $X$ be a Hausdorff space. The following are equivalent:

(a) $\left(C L(X), \tau_{F}\right)$ is Hausdorff;

(b) $\left(C L(X), \tau_{F}\right)$ is regular;

(c) $\left(C L(X), \tau_{F}\right)$ is completely regular;

(d) $X$ is locally compact.

The following theorem is the main result of our paper:

Theorem 1. Let $X$ be a Hausdorff topological space. The following are equivalent:

(1) $X$ is locally compact and Lindelöf;

(2) $\left(C L(X), \tau_{F}\right)$ is $\sigma$-compact and regular;

(3) $\left(C L(X), \tau_{F}\right)$ is Lindelöf;

(4) $\left(C L(X), \tau_{F}\right)$ is paracompact;

(5) $\left(C L(X), \tau_{F}\right)$ is normal.

Remark. When $X$ is metrizable, the conditions characterizing normality for the Fell topology also characterize metrizability [Fl], as the Lindelöf property for $X$ now is equivalent to separability and second countability.

To prove this theorem we will need the following results:

Lemma 2. Let $X$ be a Hausdorff $\sigma$-compact space. Then $\left(C L(X), \tau_{F}\right)$ is $\sigma$-compact too.

Proof. Let $\left\{K_{n}: n \in \omega\right\}$ be a sequence of compact sets in $X$ such that $X=$ $\bigcup\left\{K_{n}: n \in \omega\right\}$. Thus $C L(X)=\bigcup\left\{K_{n}^{-}: n \in \omega\right\}$. By [Be1, Exercise 5.1.4 (a)] $K_{n}^{-}$ is compact in $\left(C L(X), \tau_{F}\right)$ for every $n \in \omega$. Thus $\left(C L(X), \tau_{F}\right)$ is $\sigma$-compact.

Covers defined in the following definition have found already many applications in an investigation of hyperspaces and elsewhere.

Definition 3. Let $\kappa$ be an ordinal. An open cover $\mathcal{C}=\left\{U_{\alpha}: \alpha \in \kappa\right\}$ of $X$ is said to be well-monotone if $U_{\alpha} \varsubsetneqq U_{\beta}$ whenever $\alpha<\beta, \alpha, \beta \in \kappa$.

To any well-monotone cover $\mathcal{C}$ we will assign the subfamily $\mathcal{D}(\mathcal{C})$ of $C L(X)$ as follows. Let $\kappa$ be an ordinal such that $\mathcal{C}=\left\{U_{\alpha}: \alpha \in \kappa\right\}$. For every $\alpha \in \kappa$ put $D_{\alpha}=X \backslash \bigcup\left\{U_{\beta}: \beta<\alpha\right\}$ and set $\mathcal{D}(\mathcal{C})=\left\{D_{\alpha}: \alpha \in \kappa\right\}$.

Notice that $D_{0}=X$ and for every limit ordinal $\alpha \in \kappa$ we have $D_{\alpha}=\bigcap\left\{D_{\beta}\right.$ : $\beta<\alpha\}$.

Lemma 4. Let $\mathcal{C}$ be a well-monotone cover of a Hausdorff space $X$. Then $\mathcal{D}(\mathcal{C})$ is a closed set in $\left(C L(X), \tau_{F}\right)$.

Proof. Let $\kappa$ be an ordinal such that $\mathcal{C}=\left\{U_{\alpha}: \alpha \in \kappa\right\}$. Take $A \in C L(X) \backslash \mathcal{D}(\mathcal{C})$. Put $\alpha_{0}=\min \left\{\beta \in \kappa: D_{\beta} \not \supset A\right\}$. Recall $\bigcap\left\{D_{\beta}: \beta \in \kappa\right\}=\emptyset$ and $A \neq \emptyset$. Clearly, $\alpha_{0}$ cannot be limit, so $\alpha_{0}=\delta+1$. Hence $D_{\delta} \supset A$, so $D_{\delta} \backslash A \neq \emptyset$. Take $x \in D_{\delta} \backslash A$ and define $\mathcal{U}=\left(X \backslash D_{\alpha_{0}}\right)^{-} \cap(X \backslash\{x\})^{+}$. Then $A \in \mathcal{U}$ and $\mathcal{U} \cap \mathcal{D}(\mathcal{C})=\emptyset$.

Observe that the existence of a countable well-monotone cover of a space $X$ is a really restrictive assumption on $X$, i.e. there are spaces without any such a cover.

Lemma 5. Suppose $\mathcal{C}=\left\{U_{n}: n \in \omega\right\}$ is a well-monotone open cover of a Hausdorff space $X$. If $\left(C L(X), \tau_{F}\right)$ is normal, then $X$ is $\sigma$-compact. 
Proof. Put $\mathcal{X}=\{\{x\}: x \in X\}$. Then $\mathcal{D}(\mathcal{C})$ and $\mathcal{X}$ are disjoint $\tau_{F}$-closed sets. The normality of $\left(C L(X), \tau_{F}\right)$ implies that there are disjoint $\tau_{F}$-open sets $\mathcal{U}$ and $\mathcal{V}$ such that

$$
\mathcal{D}(\mathcal{C}) \subset \mathcal{U} \text { and } \mathcal{X} \subset \mathcal{V} \text {. }
$$

For each $D_{n} \in \mathcal{D}(\mathcal{C})$ there are open sets $W_{1}^{n}, \ldots W_{j_{n}}^{n}$ and a compact set $K_{n}$ such that

$$
D_{n} \in \bigcap\left\{\left(W_{i}^{n}\right)^{-}: i=1, \ldots, j_{n}\right\} \cap\left(\left(K_{n}\right)^{c}\right)^{+} \subseteq \mathcal{U} .
$$

We claim $X=\bigcup\left\{K_{n}: n \in \omega\right\}$. Suppose there is $z \in X \backslash \bigcup\left\{K_{n}: n \in \omega\right\} .\{z\} \in \mathcal{V}$, thus there is an open neighbourhood $P$ of $z$ and a compact set $Z$ such that

$$
\{z\} \in P^{-} \cap\left(Z^{c}\right)^{+} \subseteq \mathcal{V} .
$$

The compactness of $Z$ and $\bigcap\left\{D_{n}: n \in \omega\right\}=\emptyset$ imply that there is $m \in \omega$ with $D_{m} \cap Z=\emptyset$. Then $D_{m} \cup\{z\} \in \mathcal{U} \cap \mathcal{V}$, a contradiction.

Lemma 6. Let $X$ be a Hausdorff space and $\left(C L(X), \tau_{F}\right)$ be a normal space. There is no well-monotone cover $\mathcal{C}=\left\{U_{\alpha}: \alpha \in \kappa\right\}$ of $X$ with the cofinality of $\kappa$ greater than $\omega$.

Proof. Suppose the contrary. Again $\mathcal{D}(\mathcal{C})$ and $\mathcal{X}=\{\{x\}: x \in X\}$ are disjoint $\tau_{F}$-closed sets. The normality of $\left(C L(X), \tau_{F}\right)$ implies that there is a continuous function $f: C L(X) \rightarrow[0,1]$ such that

$$
f \nmid \mathcal{D}(\mathcal{C})=0 \text { and } f \nmid \mathcal{X}=1 .
$$

For each $n \in \omega$ take $x_{n} \in X, K_{n} \in K(X)$, an open neighbourhood $U_{n}$ of $x_{n}$ and $\lambda_{n} \in K$ such that

(1) $f\left[U_{n}^{-} \cap\left(\left(K_{n}\right)^{c}\right)^{+}\right] \subset\left(1-\frac{1}{n+2}, 1\right], x_{n} \in\left(K_{n}\right)^{c}$,

(2) $D_{\lambda_{n}} \cap\left(\bigcup\left\{K_{j} \cup\left\{x_{j}\right\}: j \leq n\right\}\right)=\emptyset$,

(3) $x_{n+1} \in D_{\lambda_{n}}, \lambda_{n+1} \geq \lambda_{n}$.

(Let $n=0$. Take $x_{0} \in X$ arbitrary. The continuity of $f$ and $f\left(\left\{x_{0}\right\}\right)=1$ implies that there are an open neighbourhood $U_{0}$ of $x_{0}$ and $K_{0} \in K(X)$ such that $x_{0} \notin K_{0}$ and $f\left[U_{0}^{-} \cap\left(\left(K_{0}\right)^{c}\right)^{+}\right] \subset\left(\frac{1}{2}, 1\right]$. Let $\lambda_{0} \in \kappa$ be such that $D_{\lambda_{0}} \cap\left(K_{0} \cup\left\{x_{0}\right\}\right)=\emptyset$. Such $\lambda_{0} \in \kappa$ always exists since $\bigcap\left\{D_{\beta}: \beta \in \kappa\right\}=\emptyset$ and $K_{0} \cup\left\{x_{0}\right\}$ is compact. Suppose now we have defined $x_{0}, x_{1}, \ldots, x_{n-1}, U_{0}, U_{1}, \ldots, U_{n-1}, K_{0}, K_{1}, \ldots, K_{n-1}$ and $\lambda_{0}, \lambda_{1}, \ldots, \lambda_{n-1}$. Take any point $x_{n} \in D_{\lambda_{n-1}}$. The continuity of $f$ and $f\left(\left\{x_{n}\right\}\right)=1$ implies the existence of $U_{n}$ and $K_{n}$ which verifies 1). There is $\lambda_{n} \in \kappa$ with $\lambda_{n}>$ $\lambda_{n-1}$ and $D_{\lambda_{n}} \cap\left(\bigcup\left\{K_{j} \cup\left\{x_{j}\right\}: j \leqq n\right\}\right)=\emptyset$. $)$

Put $\lambda=\sup \left\{\lambda_{n}: n \in \omega\right\}$. Then $\lambda \in \kappa$ and $D_{\lambda}=\bigcap\left\{D_{\lambda_{n}}: n \in \omega\right\}$. For every $n \in$ $\omega$ put $L_{n}=D_{\lambda_{n}} \cup\left\{x_{n}\right\}$. So $f\left(L_{n}\right) \in\left(1-\frac{1}{n+2}, 1\right]$ by 1$)$, since $L_{n} \in\left(U_{n}\right)^{-} \cap\left(\left(K_{n}\right)^{c}\right)^{+}$. Let $C \in K(X)$ be such that $C \cap D_{\lambda}=\emptyset$. Then there is $n \in \omega$ with $D_{\lambda_{n}} \cap C=\emptyset$. So $\left\{L_{n}: n \in \omega\right\} \tau_{F}$-converges to $D_{\lambda}$, which contradicts $f\left(D_{\lambda}\right)=0$.

Theorem 7. Let $X$ be a Hausdorff space and $\left(C L(X), \tau_{F}\right)$ be a normal space. Then $X$ is a Lindelöf space.

Proof. Suppose $X$ is not Lindelöf. In the family of all open covers of $X$ without any countable subcover there is an open cover $\mathcal{G}$ of $X$ with the minimal cardinality $|\mathcal{G}|$. Let $\kappa$ be the first ordinal having the cardinality $|\mathcal{G}|$. Thus $\mathcal{G}=\left\{U_{\lambda}: \lambda<\kappa\right\}$. 
For every $\lambda<\kappa$ define $R_{\lambda}=\bigcup\left\{U_{\xi}: \xi \leqq \lambda\right\}$. Then $R_{\lambda} \neq X$ for each $\lambda<\kappa$. There is a subfamily $\left\{R_{\lambda_{\gamma}}: \gamma<\kappa\right\}$ of $\left\{R_{\lambda}: \lambda<\kappa\right\}$ which is a well-monotone cover of $X$. (By transfinite induction we define a sequence $\left\{\lambda_{\gamma}: \gamma<\kappa\right\} \subset[0, \kappa)$ in the following manner. Let $\lambda_{0}=0$. Having defined $\lambda_{\gamma}$ let $\lambda_{\gamma+1}$ be the first $\lambda>\lambda_{\gamma}$ such that $R_{\lambda_{\gamma}}$ is properly contained in $R_{\lambda}$. For $\gamma$ a limit ordinal, let $\lambda_{\gamma}=\sup \left\{\lambda_{\beta}: \beta<\gamma\right\}$. If $\sup \left\{\lambda_{\beta}: \beta<\gamma\right\}=\kappa$, then $\left\{R_{\lambda_{\beta}}: \beta<\gamma\right\}$ is an open cover of $X$ with the cardinality smaller than $|\mathcal{G}|$, thus there must exist a countable subcover of $\left\{R_{\lambda_{\beta}}: \beta<\gamma\right\}$ which leads to a contradiction by Lemma 5 . Thus $\alpha_{\gamma}<\kappa$.)

For every $\gamma<\kappa$ put $P_{\gamma}=R_{\lambda_{\gamma}}$. Thus $\left\{P_{\gamma}: \gamma<\kappa\right\}$ is a well-monotone cover of $X$. Thus by Lemma $6, \kappa$ is cofinal with $\omega$. Take $\kappa_{n} \nearrow \kappa, n \in \omega$. Define

$$
W_{n}=\bigcup\left\{P_{c}: c \leqq \kappa_{n}\right\} .
$$

Then $\left\{W_{n}: n \in \omega\right\}$ is a well-monotone cover of $X$. Hence $X$ is $\sigma$-compact by Lemma 5, a contradiction.

Proof of Theorem 1. (1) $\Rightarrow$ (2) The local compactness of $X$ implies the regularity of $\left(C L(X), \tau_{F}\right)$ by Proposition A. If $X$ is locally compact and Lindelöf, then $X$ is $\sigma$-compact. By Lemma $2\left(C L(X), \tau_{F}\right)$ is $\sigma$-compact.

$(2) \Rightarrow(3),(3) \Rightarrow(4)$ and $(4) \Rightarrow(5)$ are clear.

$(5) \Rightarrow(1)$ If $\left(C L(X), \tau_{F}\right)$ is normal, then $X$ is Lindelöf by Theorem 7 . If $X$ is Hausdorff, then $\left(C L(X), \tau_{F}\right)$ is $T_{1}$. Thus the normality of $\left(C L(X), \tau_{F}\right)$ implies its regularity, which gives us the local compactness of $X$ by Proposition A.

At the end of this paper notice that the first countability of the Fell topology implies its normality. By a result in [HL] $\left(C L(X), \tau_{F}\right)$ is first countable if and only if $X$ is first countable, hereditarily separable, and each open set is hemicompact (see also [Be2]). The hemicompactness of $X$ together with the first countability of $X$ imply that $X$ is locally compact and Lindelöf.

\section{REFERENCES}

[At] H. Attouch, Variational convergence for functions and operators, Pitman, Boston, 1984. MR 86f: 49002

[Be1] G. Beer, Topologies on Closed and Closed Convex Sets, Kluwer Academic Publishers, 1993. MR 95k:49001

[Be2] G. Beer, On the Fell topology, Set-Valued Analysis 1 (1993), 69-80. MR 95a:54024

[BT] G. Beer, R.Tamaki, The infimal value functional and the uniformization of hit-and-miss hyperspace topologies, Proc. Amer. Math. Soc. 122 (1994), 601-611. MR 95a:54023

[En] R.Engelking, General Topology, PWN Warszawa, 1977. MR 58:18316b

[Fe] J.Fell, A Hausdorff topology for the closed subsets of a locally compact non-Hausdorff space, Proc.Amer.Math.Soc. 13 (1962), 472-476. MR 25:2573

[Fl] J.Flachsmeyer, Verschiedene Topologisierungen im Raum der abgeschlossenen Teilmengen, Math. Nachr. 26 (1964), 321-337. MR 30:4233

[HL] L.Hola, S.Levi, Decomposition properties of hyperspace topologies, Set-Valued Analysis to appear.

[Ke1] J.Keesling, Normality and properties related to compactness in hyperspaces, Proc. Amer. Math. Soc. 24 (1970), 760-766. MR 40:6507

[Ke2] J.Keesling, On the equivalence of normality and compactness in hyperspaces, Pacific J. Math. 33 (1970), 657-667. MR 42:2418

[Ma] G. Matheron, Random sets and integral geometry, Wiley, New York, 1975. MR 52:6828

[Mi] E.Michael, Topologies on spaces of subsets, Trans. Amer.Math. Soc. 71 (1951), 152-182. 
[Po] H.Poppe, Einige Bemerkungen uber der raum der abgeschlossen mengen, Fund. Math. 59 (1966), 159-169. MR 33:6573

[Ve] N.H. Velicko, On the space of closed subsets, Sibirsk.Math.Z. 16 (1975), 627-629. (Russian; English translation: Siberian Math. J. 16 (1975), 484-486). MR 51:13969

Mathematical Institute, Slovak Academy of Sciences, Štefániková 49 Bratislava, SLOVAKIA

E-mail address: hola@mau.savba.sk

Dipartimento di Matematica, Universita di Milano, Via C. Saldini 50, 20133 Milano, ITALY

E-mail address: slevi@vmimat.mat.unimi.it

Mathematical Institute, Czech Academy of Sciences, Žitná 25, 11567 Praha, Czech REPUBLIC

E-mail address: pelant@beba.cesnet.cz 